\title{
WHERE IS THE ROTATION CENTRE OF THE LMC?
}

\author{
R. WIELEBINSKI \\ Max-Planck-Institut für Radioastronomie \\ Auf dem Hügel 69 \\ D-5300 Bonn 1 \\ F.R. Germany \\ E. BAJAJA \\ Instituto Argentino de Radioastronomia \\ Casilla de Correo No. 5 \\ 1894 Villa Elisa (Prov. de Bs. As.) \\ Argentina
}

\begin{abstract}
The structure of the Large Magellanic Cloud (LMC) has posed many questions and has led to many controversial discussions. Although classified as an 'irregular', repeated claims have been made suggesting that the LMC has spiral structure. Furthermore, this 'spiral' structure seems to originate in the 30-Doradus nebula which, as a result, was sometimes referred to as the 'nucleus' of the LMC. We summarize the recent data on the LMC and re-examine the HI data to point out that possibly different rotation centres exist for different components.
\end{abstract}

\section{Introduction}

An extensive review of data available for the LMC was collected by de Vaucouleurs and Freeman (1972). In their Table 2 a listing of the centroids of the various components is given. One of the curious aspects of these data was the fact that no nucleus-like source was found near the positions of the (mainly optical) centroids. More recent reviews of the LMC data by Westerlund (1989, 1990) confirm this surprising result.

In recent years much new data have been collected, especially on the 'young' components in the LMC. New radio continuum surveys (see Klein et al. 1990) have been made with polarization information. Full maps of the LMC have been published in the FIR and in the X-ray range. All these data, which delineate either molecular gas, the dust or the relativistic component (magnetic fields) of the LMC, seem to indicate a unique function of 30 Dor. In all these spectral ranges filaments (spiral arms?) originate in 30 Dor and can be traced up to some kpc away.

\section{The HI data}

The one additional data base which should help us to study the morphology of the LMC is the HI data base. The earlier work of McGee and Milton (1966) was supplemented by newer observations of Rohlfs et al. (1984) and Rohlfs (1990). It must be noted, however, that the HI data cover only the inner parts of the LMC: usually the HI envelope of a galaxy is much larger than either that delineated by radio continuum emission or the optical image. The HI total intensity has many similarities to the other 'young' components. Rohlfs et al. (1984) have determined a 
rotation curve for the $\mathrm{LMC}$ for the PA equal to $208^{\circ}$ relative to the optical centre (Fig. 1). Note that the rotation centre which was determined by 'symmetrization by eye' lies at $\mathrm{X}^{\prime}=0^{\circ} .55$.

\section{Rotation curves}

We have been studying numerous nearby galaxies in $\mathrm{CO}$ with the 30-m telescope of IRAM at Pico Veleta and with the 15-m SEST dish at La Silla. In all our studies a very characteristic rotation curve is found: the rigid rotation around the nucleus, two peaks, followed by a transfer to differential rotation. As an example the rotation curve of M82 is shown in Fig. 2. Such curves are found not only for the edge-on galaxies but also, after correction for inclination, for the more faceon systems. In fact, based on our experience, we would set the rotation centre of the HI data (in Fig. 1) at $X^{\prime}=-1^{\circ} .5$ which places it very near 30 Dor.

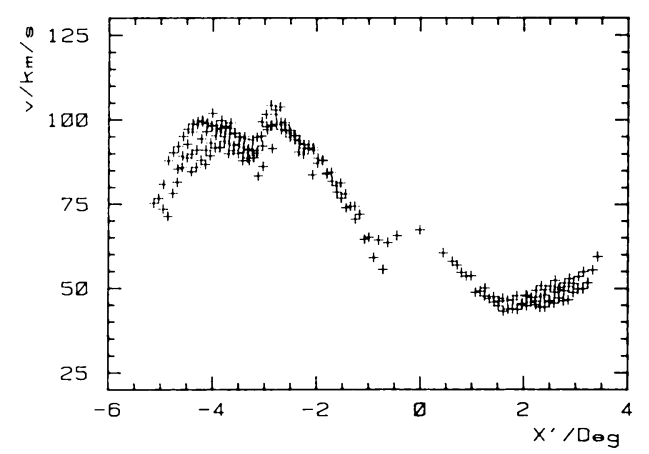

Figure 1. The HI rotation curve for the LMC from Rohlfs et al. (1984). Note $1^{\circ} \simeq 1$ kpc.

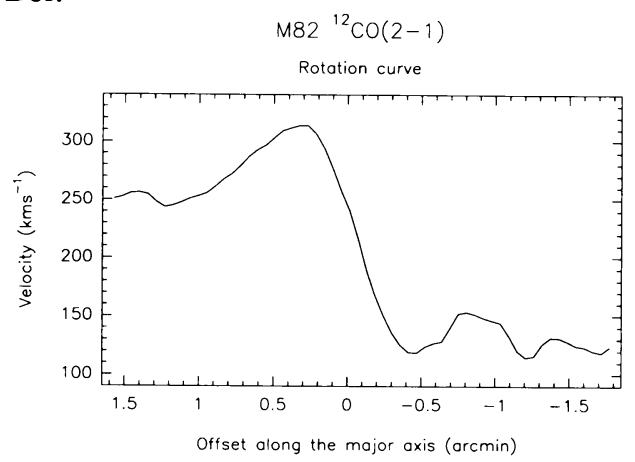

Figure 2. A rotation curve of M82 from $\mathrm{CO}(2-1)$ data. Note $1^{\prime} \simeq 1 \mathrm{kpc}$.

We have reanalyzed the HI data of Rohlfs et al. (1984). We assumed the rotation centre to be the 30-Dor nebula and constructed rotation curves for various position angles. The rotation curve for PA equal to $180^{\circ}$ is shown in Fig. 3. The HI data are consistent with the rotation of the neutral gas around 30-Dor nebula.

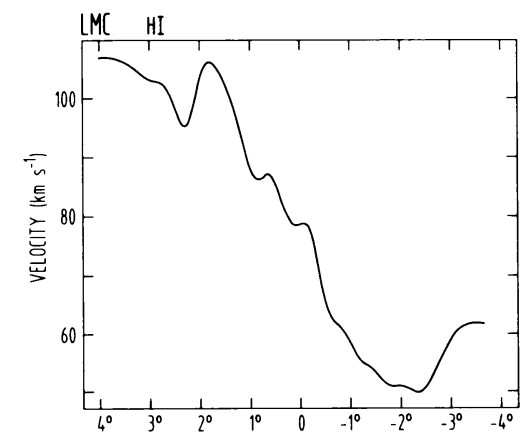

Figure 3. A rotation curve determined from the HI data of Rohlfs et al. (1984) but with 30 Dor as the centre. The cut is with $\mathrm{PA}=180^{\circ}$. Note $1^{\circ} \simeq 1 \mathrm{kpc}$. 


\section{The 30-Doradus 'Nucleus'}

The 30-Dor nebula was found to be 'entirely thermal' by Mills et al. (1978) based on multifrequency radio studies. We wish to point out that our Galactic centre was consistently interpreted to be thermal (e.g. Genzel \& Townes, 1987) until high linear polarization was found (Seiradakis $e t$ al. 1985). A multi-frequency study of the Galactic centre (Reich et al. 1988) showed that there is considerable nonthermal emission but with flat, or even positive spectral index. Indeed, the 30Dor nebula shows many properties which we usually associate with a nucleus of a galaxy. In particular, the filamentary ridges which originate in 30 Dor suggest a nuclear source. A detection of a compact radio source (VLBI observations) could decide this discussion but may require mJyVLBI, still some time away in the southern skies.

\section{Discussion}

If we accept that HI gas rotates around the 30-Dor nebula, we must ask: is it possible for various components of the galaxy to rotate around different centres? Certainly the interaction between the LMC, SMC and the Milky Way must be considered in this context. The models of Murai and Fujimoto (1980) and Fujimoto (1990) suggest that the collision of the SMC with the LMC could lead to such an extreme situation. Theoretical models certainly are required to study such possibilities. Also, new observational material is needed for both the 30-Dor nebula and the whole galaxy.

\section{Acknowledgment}

We thank Prof. K. Rohlfs for making the HI data available to us in machine-readable form.

\section{References}

de Vaucouleurs, G., Freeman, K.C. (1972), Vistas in Astronomy 14, 163.

Fujimoto, M. (1990), these proceedings.

Genzel, R., Townes, C.H. (1987), Ann. Rev. Astron. Astrophys. 25, 377.

Klein, U. et al. (1990), these proceedings.

McGee, R.X., Milton, J.A. (1966), Aust. J. Phys. 19, 343.

Mills, B.Y., Turtle, A.J., Watkinson, A. (1978), M.N.R.A.S. 185, 263.

Murai, T., Fujimoto, M. (1980), Publ. Astron. Soc. Japan 32, 581.

Reich, W., Sofue, Y., Wielebinski, R., Seiradakis, J.H. (1988), Astron. Astrophys. 191, 303.

Rohlfs, K. (1990), these proceedings.

Rohlfs, K., Kreitschmann, J., Siegman, B.C., Feitzinger, J.V. (1984), Astron. Astrophys. 137, 343.

Seiradakis, J.H., Lasenby, A.N., Yusef-Zadeh, F., Wielebinski, R., Klein, U. (1985), Nature 317, 697.

Westerlund, B.E. (1989), Recent Developments of Magellanic Cloud Research, K.S. de Boer et al. (eds.) (Observatoire de Paris) p.159.

Westerlund, B.E. (1990), these proceedings. 